\title{
Erratum to: Hyperhomocysteinemia and Related Genetic Polymorphisms Correlate with Ulcerative Colitis in Chinese Han population in Central China
}

\author{
Yi Jiang $\cdot$ Xuanping Xia $\cdot$ Wenxing Wang $\cdot$ Limiao Lin \\ Changlong Xu - Zhenzai Cai $\cdot$ Bo Zheng $\cdot$ Jihua Pei \\ Sujian Shen $\cdot$ Bing Xia
}

Published online: 19 January 2012

(C) Springer Science+Business Media, LLC 2012

Erratum to: Cell Biochem Biophys (2012) 62:203-210

DOI 10.1007/s12013-011-9283-4

Unfortunately the title of the article was furnished incorrectly in the original publication of the article. The population and UC patients should be Chinese Han population in Central China, not Southeast China. Therefore, the title of the article should be "Hyperhomocysteinemia and Related Genetic Polymorphisms Correlate with Ulcerative Colitis in Chinese Han population in Central China", and related term in the text should be corrected.

Moreover, the affiliation and email address of the corresponding author, Prof. Bing Xia, is corrected in this erratum.

The online version of the original article can be found under doi:10.1007/s12013-011-9283-4.

Y. Jiang · B. Xia $(\bowtie)$

Department of Gastroenterology, Zhongnan Hospital

of Wuhan University School of Medicine, Wuhan 430071,

People's Republic of China

e-mail: bingxia2004@yahoo.com.cn

X. Xia $\cdot$ W. Wang $\cdot$ L. Lin $\cdot$ C. Xu $\cdot$ Z. Cai

B. Zheng $\cdot$ J. Pei $\cdot$ S. Shen

Department of Gastroenterology, Second Affiliated Hospital

of Wenzhou Medical College, Wenzhou 325000,

People's Republic of China 